\title{
Managing the Competencies of Team Members in Design Projects through Multi-period Task Assignment
}

\author{
Onanong Hlaoittinun, Eric Bonjour, and Maryvonne Dulmet \\ Institute FEMTO-ST UMR CNRS 6174, Department AS2M \\ 24, Rue Alain Savary, 25000 Besançon, France \\ \{onanong.hlaoittinun, eric.bonjour, maryvonne.dulmet\} @ens $2 \mathrm{~m}$. fr
}

\begin{abstract}
Product design projects involve multiple team members with various knowledge and competencies that have to evolve with time, due to rapid organizational, technological and marketing changes. Project managers require management methods to optimize the assignment of tasks to human resources according to their competency levels and the need for competency evolution. In this paper, we tackle this optimization problem in the context of multiple design projects and multiple periods. The model we propose seeks to minimize two function objectives: (i) minimize the supplementary salary cost due to the extended task duration when using under-competent employees and (ii) minimize the financial penalties when the competency goals have not been reached. This problem has been solved by means of a simulated annealing -based optimization algorithm.
\end{abstract}

Keywords: Project management, Design project, Task assignment, Multiskilled Resource allocation, Competency modeling.

\section{Introduction}

In product development projects, project managers face highly complex situations in which they have to deal with a great number of components with their interactions, a multiplicity of design tasks with their interdependencies, and a great number of team members with their evolution in competencies. They have to make difficult decisions concerning the allocation of numerous team members to design tasks.

The rapid evolution of technology in product design and business process reengineering often results in a change in the definition of design tasks. Consequently, R\&D departments can suffer from a lack of competencies and may be unable to design future innovative products. In this paper, we are interested in tackling the problem of managing the team members' competencies and their evolution during the execution of design projects. This issue becomes one of the greatest concerns of project managers.

It has been commonly accepted that an appropriate task assignment can either maintain or improve the competency level of the team member assigned to the corresponding task. So project managers need methods to help them to optimize task assignments and to ensure that the evolution of team members' competencies is relevant to the objectives of future projects. 
Research works concerning task assignment problem can be classified according to two criteria: "single-period" or "multi-period" task assignment and "static" or "dynamic" competency modeling. "Single-period" task assignment refers to the situation in which all tasks are considered as a single group, in other words, when time constraints between tasks have not been taken into account. "Multi-period" task assignment concerns the situation in which precedence constraints have been considered. "Static" competency modeling represents the situation in which the levels of competency for each actor are unchanged during the execution of tasks. "Dynamic" competency modeling aims at representing and simulating the evolution of the competency level for each actor during the execution of tasks.

These criteria, when combined, help us to divide related works into four categories.

The first category concerns "mono-period" task assignment with "static" modeling of competency. This is the easiest case because there is a single group of tasks to be assigned and competency levels are unvaried during the task execution.

The second category concerns "mono-period" task assignment with "dynamic" competency modeling [1]. This category concerns the evolution of competency levels but these competency levels are measured and followed during only one period (at the beginning and at the end). So, this task assignment model is not aimed to plan competency levels of actors in the long term. However, it needs incorporating learning and forgetting curves in the decision model.

The third category deals with "multi-period" task assignment with "static" competency modeling [2-3]. In project scheduling problems, this problem is called MSPSP which stands for Multi-skill Project Scheduling Problem. For instance, BellenguezMorineau [2] proposes a method that solves the MSPSP problem and ensures that the assigned actors are competent enough to carry out project tasks. This method seeks to minimize the total completion time of the project (Cmax).

The fourth category concerns "multi-period" task assignment with "dynamic" competency modeling [4-5]. The authors propose a model that optimizes the long term resource allocation. The evolution of competency (learning effect and knowledge depreciation) is also considered. The authors assume that the competency level of the assigned person will increase in accordance to the number of periods he/she activates this competency and his/her learning rate. Similarly, the competency level of a particular actor will decline in accordance to a knowledge depreciation rate if this actor has not been assigned to tasks corresponding to this competency. However, the authors have not modeled design project environments [4] or they have not focused on competency-based objectives [5].

Because of the increasing importance of competency evolution in the field of project management and due to a lack of research works concerning this issue, we are interested, in this paper, in dealing with the latter type of problem described above.

In competency modeling, Harzallah et al. suggests the CRAI model (Competency, Resource, Aspect, Individual), associated with axioms based on set theory [6]. Competencies are characterized by sets of knowledge, know-how and behavior associated to a context and linked to individual actors. Based on a classical evaluation of the "knowledge", "know-how" and "behavior" characteristics, a mathematical aggregation is suggested to provide a quantitative evaluation of competencies. Boucher et al. [7] propose a literature review of competency management from a performance point of view. They underline that the characterization of competency is a first step for modeling the development of competency and improving goals of competency management. 
Different attributes of competency have been used in the field of team formation to model competency, such as qualification (0 or 1) [8], technical knowledge, skills and know-how [9-10], psycho-sociological capacities or personal traits [11]. In the rest of this paper, to simplify the terminology, the concept of knowledge will cover all these attributes.

The organization of this paper is as follows: Section 2 presents the main steps of the task assignment modeling we propose. Section 3 outlines the mathematical formulation and the optimization approach for the considered problem. To illustrate the interests of this approach, simulation results are presented and shortly discussed in section 4. Section 5 sums up the paper and opens new directions of research.

\section{Proposition for Modeling Multi-period Task Assignment}

The method we propose is decomposed into four main stages of modeling that will be shortly outlined in this part.

\subsection{Identifying Relevant Knowledge to Characterize Tasks and Actors}

We define a generic set of tasks, indexed by $i(i=1, \ldots, \mathrm{M})$, a set of projects, indexed by $l(l=1, \ldots, \mathrm{P})$, a set of periods, indexed by $k,(k=1, \ldots, \mathrm{K})$, a set of actors, indexed by $j(j=1, \ldots, \mathrm{N})$ and a set of knowledge, indexed by $c,(c=1, \ldots, \mathrm{C})$.

We can classify two types of tasks used in multi-period task planning: generic and specific tasks. Each generic task $T_{i}$ corresponds to one competency and covers a class of specific tasks $T_{i}^{k, l}$ sharing the same knowledge but with different performance levels, depending on period $k$ and project $l$.

Task characterization. Performance levels concerning a task can be characterized by two types of expertise levels: competency level and knowledge level. The variable $r q_{i}^{k, l}$ represents the competency level required by specific task $T_{i}^{k, l}$. The variable $r_{i, c}^{k, l}$ represents the performance level in knowledge $c$ required by specific $\operatorname{task} T_{i}^{k, l}$. We assume that $r_{i, c}^{k, l}=r q_{i}^{k, l} \times r_{i, c}$, where $r_{i, c}$ is the performance level in knowledge $c$ required by the generic task $i$.

Actor characterization. The variable $r_{2}{ }_{c, j}^{k}$ represents the performance level in knowledge $c$ that is acquired by actor $j$ in period $k$. We assume that the performance levels in knowledge $c$ evolve during the task execution and depend on period $k$.

\subsection{Calculating the "A priori" Compatibility Level and the Work Efficiency}

The compatibility level aims at estimating the similarity between specific task $T_{i}^{k, l}$ and actor $j$ before the assignment of tasks in period $k$. This similarity is computed for each actor $j$ by penalizing his/her insufficient performance level for each type of knowledge according to the performance level required by $T_{i}^{k, l}$ (Equation 1). This indicator has been presented in detail in [12]. The value of this indicator is included between 0 and 1 . 


$$
v_{i, j}^{k, l}=r_{i, \bullet}^{k, l} \circ r_{\bullet, j}^{k-1}=1-\frac{\sum_{c=1}^{O} \max \left(0, r_{i, c}^{k, l}-r r_{c, j}^{k-1}\right)}{\sum_{c=1}^{O} r_{1_{i, c}^{k}}^{k, l}}, 0 \leq v_{i, j}^{k, l} \leq 1 .
$$

The compatibility level helps to select the appropriate actor for the $k^{\text {th }}$ assignment by turning it into the coefficient of work efficiency. We suppose that the time spent on a task has to be adjusted according to the "a priori" compatibility level $v_{i, j}^{k, l}$. Therefore, there will be additional costs due to the assignment of specific task $T_{i}^{k, l}$ to under-competent actor $j$. We introduce the coefficient of work efficiency $\gamma_{i, j}^{k, l}$ (Equation 2 - note that $\gamma_{i, j}^{k, l}=1$ if $v_{i, j}^{k, l}=1$, that is, if the actor is competent). Then this coefficient is used to compute the actual execution time of specific task $T_{i}^{k, l}$ for actor $j: \gamma_{i, j}^{k, l} \times L_{i}^{k, l}$, where the theoretical time $L_{i}^{k, l}$ is given. This indicator is explained in detail in [13].

$$
\gamma_{i, j}^{k, l}=2-v_{i, j}^{k, l} .
$$

\subsection{Propagating the Evolution of Competency after Each Period of Task Assignment}

We assume that if actor $A_{j}$ is allocated to task $T_{i}^{k, l}$ (that is, $x_{i, j}^{k, l}>0$ ), this actor either maintains his/her performance level in knowledge $c$ if $r_{1, c}^{k, l} \geq r_{c, j}^{k}$ or develops this performance level in knowledge $c$ if $r_{i, c}^{k, l}<r_{c, j}^{k}$. If actor $j$ is not allocated to task $T_{i}^{k, l}$ (that is, $x_{i, j}^{k, l}=0$ ), his/her performance level in knowledge $c$ will decrease. To shorten this paper, we have not presented in detail how we have modeled the competency evolution.

\subsection{Calculating the "A posteriori” Compatibility Level (Competency Level)}

The variable $r 3_{i, j}^{k}$ estimates the competency level of actor $j$ for generic task $T_{i}$ (that is, the compatibility between actor $j$ and generic task $T_{i}$ ). This variable is calculated at the end of period $k$ when actor $j$ has carried out the tasks he/she has been responsible for.

$$
r_{i, j}^{k}=r_{i, \bullet} \circ r_{\bullet, j}^{k}=1-\frac{\sum_{c=1}^{O} \max \left(0, r_{i, c}-r_{c, j}^{k}\right)}{\sum_{c=1}^{O} r_{1_{i, c}}}, 0 \leq r 3_{i, j}^{k} \leq 1 .
$$

\section{Mathematical Formulation for the Task Assignment Problem}

The proposed model is based on the following assumptions:

- An under-competent actor could be assigned to a task but he/she will be accompanied by a trainer (or teaching tutor), which will generate additional costs to the project.

- $\quad$ Each assigned task is supposed to be carried out with success. 
- There is no load constraint for tutors

- The actor's capacity can be estimated in any time unit (day, month, etc). This capacity is supposed to exclude all the absence time due to the unavailability of employees (planned/unplanned absence, legal and social constraints, etc.)

The overall objective function, the cost functions and the constraints that are used in the proposed task assignment model are described as follows:

$$
\begin{aligned}
& \operatorname{Min}\left\{\left[\sum_{k=1}^{K} \sum_{l=1}^{P} \sum_{i=1}^{M} \sum_{j=1}^{N}\left[\left(\gamma_{i, j}^{k, l} \times L_{i}^{k, l}\right) \times S_{j}+\left(\gamma_{i, j}^{k, l}-1\right) \times L_{i}^{k, l} \times S T\right] x_{i, j}^{k, l}\right]+\sum_{i=1}^{M} \varphi_{i}\left(O_{i}-R_{i}\right)\right\} . \\
& \sum_{j=1}^{N} x_{i, j}^{k, l}=1, \forall i=1, \ldots M, \forall l=1, \ldots, P, \forall k=1, \ldots, K \\
& \sum_{i=1}^{M} \sum_{l=1}^{P} \gamma_{i, j}^{k, l} L_{i}^{k, l} x_{i, j}^{k, l} \leq L M_{j, k}, \forall j=1, \ldots N, \forall k=1, \ldots K \\
& 0 \leq x_{i, j}^{k, l} \leq 1, \forall i, j, k, l ; \quad x_{i, j}^{k, l} \in R^{+}
\end{aligned}
$$

The optimization of the assignment problem aims at minimizing the total project costs by determining the best assignment of tasks to actors. The overall objective function is split up into three criteria (or kinds of cost): the actors' salary, the tutors' salary and the financial penalties due to a lack of competent actors at the end of the horizon.

Criterion related to actors' salary $\left[\left(\gamma_{i, j}^{k, l} \times L_{i}^{k, l}\right) \times S_{j}\right]$ This criterion relates to the competency level of employees concerning the assigned tasks. It is calculated from their salary rates and their estimated work duration (based on the coefficient of work efficiency). The product of the coefficient of work efficiency and the theoretical work duration $\left(\gamma_{i, j}^{k, l} \times L_{i}^{k, l}\right)$ represents an estimated work duration for specific task $T_{i}^{k, l}$ of actor $j$. This duration is longer than the theoretical work duration $\left(L_{i}^{k, l}\right)$ if the person is under competent $\left(\gamma_{i, j}^{k, l}>1\right)$ for specific tasks.

Criterion related to tutors' salary $\left[\left(\gamma_{i, j}^{k, l}-1\right) \times L_{i}^{k, l} \times S T\right]$ This criterion is derived from the case when tutors have to help the under-competent actor to accomplish the assigned tasks. This cost is based on the tutoring duration (calculated from the coefficient of work efficiency) and the salary rate of tutors.

Criterion related to financial penalties $\sum_{i} \varphi_{i}\left(O_{i}-R_{i}\right.$ These penalties are explained as lost incomes that companies could have if their competency objectives have not been reached. Competency objectives $O_{i}(i=1, \ldots, \mathrm{M})$ are used to describe the number of expected competent actors, required by generic tasks. To determine whether a person is competent, we define required performance thresholds, called thres $i$ ( $i=$ $1, \ldots, \mathrm{M})$. Actor $j$ is judged competent for task $i$ if $r_{3}{ }_{i, j}^{k} \geq$ thres $s_{i}$. At the end of the horizon of task assignment $(k=\mathrm{K})$, we have obtained the number of competent actors $R_{i}$ for each task $i(i=1, \ldots, \mathrm{M}):{ }_{R_{i}}=\sum_{j=1}^{N} \max \left(0 ; E\left[r_{3, j}^{K}-\right.\right.$ thres $\left.\left._{i}+1\right]\right)$. The penalty rate for each missing competent actor is defined by constant $\varphi_{i}$. 
Two types of constraints are used in this model.

Workload constraint (Equation 4) This constraint aims at ensuring that each task is completely assigned.

Resource load constraint (Equation 5) This constraint aims at ensuring that the total assigned load concerning an actor does not exceed his/her capacity.

To solve this problem, we have to face two difficulties: the non-linearity in competency evolution and the objectives in competency development at the end of the horizon of task assignment. To deal with these difficulties, this N-P hard problem can not be solved by the known exact optimization methods. Therefore, we develop a metaheuristic approach to solve the problem. Simulated annealing (SA) is a generic probabilistic meta-algorithm for the overall optimization problem, namely locating a good approximation to the minimum of a given function in a large search space [14-15].

The choice of using the simulated annealing is justified by the fact that we are able to find an initial solution to the global problem and to generate neighbors. The initial solution of this task assignment problem is found by considering $k$ sub-problem separately (for each period $k$ ). The optimal solution of each sub-problem is found by using the linear programming successively for each period $k$ and by propagating the evolution of competency (the algorithm starts from $k=1, \ldots, \mathrm{K}$ ). The parameter setting of SA (such as neighbor function, acceptance probabilities, annealing schedule) is described in detail in [13]. When the SA algorithm reaches the stop condition, it returns the final assignment solution.

\section{Computational Results}

The case study concerned two projects that have been planned over three periods. The lists of 15 generic tasks, 20 actors and 23 types of knowledge have been generated. The performance levels in knowledge have been defined for each task and each actor. The project managers are in charge of defining these data for their projects. To shorten this paper, we sum up the results according to two viewpoints: competencybased aspect and financial aspect. For more information, please see [13].

\subsection{Competency-Based Viewpoint}

Figure 1-(1) shows the variation of performance levels in knowledge acquired by actor $\mathrm{N}^{\circ} 1$ after 3 periods of assignment (initial period, $k=0$; last period, $k=3$ ). Figure $1-(2)$ shows the variation of competency levels (concerning generic tasks) acquired by actor $\mathrm{N}^{\circ} 1$ after each period. These results confirm that knowledge and competencies that have been activated to carry out tasks have been developed. The performance levels in knowledge that have not been mobilized in order to carry out tasks have declined.

\subsection{Financial Viewpoint}

By using the simulated annealing based algorithm, the total cost obtained by the partial optimization of the $\mathrm{k}$ sub-problems has been decreased about $3 \%$. The variation of the total cost is presented by the first graph in Figure 2, and the variation of three sub-costs is presented by the other graphs. Concerning the financial penalty, we found that, before task assignment $(\mathrm{k}=0), 9$ competent actors were missing. At the end of the horizon, the number of competent actors was increased and only 5 competent actors were still missing. 


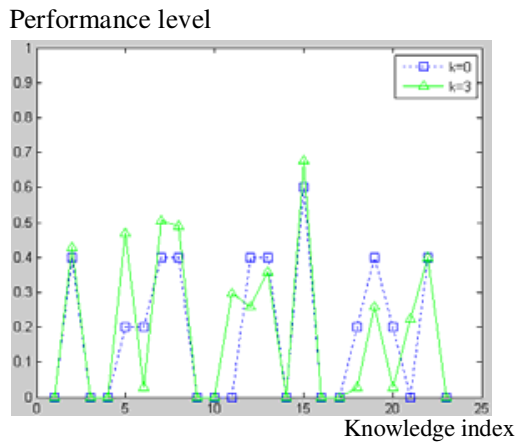

(1)

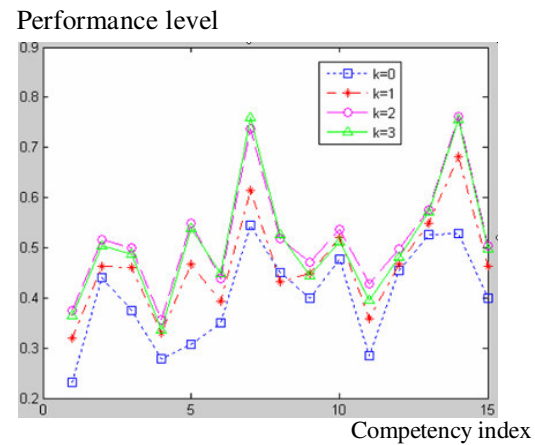

(2)

Fig. 1. Evolutions of the performance levels in knowledge and in competency

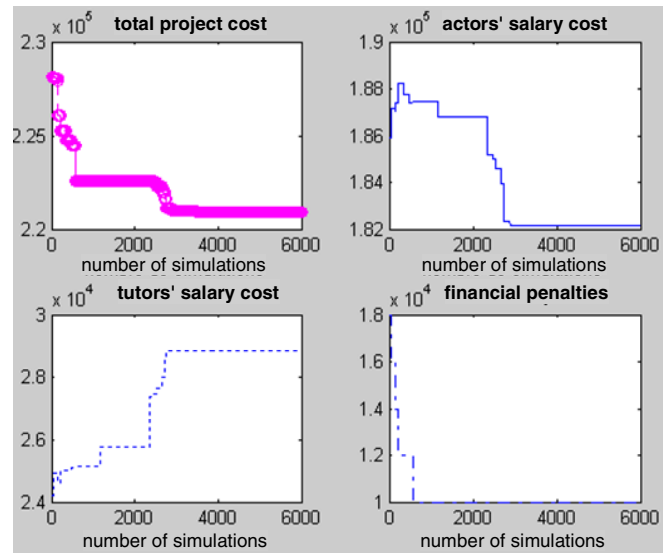

Fig. 2. Financial viewpoint

\section{Conclusion}

In this article, we have proposed a model of the multi-period task assignment problem with the modeling of the evolution of competencies. The short literature review that we have presented reveals that this problem has been rarely studied. This NP-hard problem has been solved by a simulated annealing -based optimization algorithm. The main assumption of this model is justified by psychological studies: an actor develops his/her knowledge when he/she has to carry out the task that activates this knowledge. Two types of tasks have been defined: generic tasks for modeling competencies and specific tasks for modeling their occurrences. Tasks have been characterized according to two levels: performance level in knowledge and competency level. A compatibility indicator between tasks and actors has also been proposed in order to calculate the coefficient of work efficiency that is used to penalize under-competent actors in the assignment problem.

The obtained results confirm that this method is convincing from the financial and competency-based viewpoints. Globally, we decreased the project costs, even if this decrease seems to be rather low. On the one hand, this can be explained by the fact that this decrease depends on how we fix the penalty rate. On the other hand, the 
initial solution (obtained by linear programming) is a good solution. From the competency-based viewpoint, the increase in the number of competent actors after the horizon of task assignment has proved that the proposed algorithm tried to reach the objectives of competency development.

Further work will concern the sensitivity analysis of the proposed algorithm. Project managers' estimations concerning the performance level in knowledge and the competency level that is required by a specific task may be imprecise. Due to the way of computing the compatibility level (Equation 1), we have good reasons to assume that the proposed method is few sensitive. Other experimentations will be led to prove this point.

\section{References}

1. Sayin, S., Karabati, S.: Assigning cross-trained workers to departments: A two-stage optimization model to maximize utility and skill improvement. European Journal of Operational Research 176(3), 1643-1658 (2007)

2. Bellenguez, O., Néron, E.: Lower Bounds for the Multi-Skill Project Scheduling Problem with Hierarchical Levels of Skill. In: Burke, E.K., Trick, M.A. (eds.) PATAT 2004. LNCS, vol. 3616, pp. 229-243. Springer, Heidelberg (2005)

3. Corominas, A., Pastor, R., Rodriguez, E.: Rotational allocation of tasks to multifunctional workers in a service industry. International Journal of Production Economics 103, 3-9 (2006)

4. Gutjahr, W.J., Katzensteiner, S., Reiter, P., Stummer, C., Denk, M.: Competence-driven project portfolio selection, scheduling and staff assignment. Central European Journal of Operations Research 16(3), 281-306 (2008)

5. Fowler, J.W., Wirojanagud, P., Gel, E.S.: Heuristics for workforce planning with worker differences. European Journal of Operational Research 190, 724-740 (2008)

6. Harzallah, M., Berio, G., Vernadat, F.: Analysis and modeling of individual competencies: toward better management of human resources. IEEE Transactions on Systems, man, and Cybernetics-PART A: Systems and Humans 36(1) (2006)

7. Boucher, X., Bonjour, E., Grabot, B.: Formalisation and use of competencies for industrial performance optimisation: a survey. Computers in industry 58(2), 98-117 (2007)

8. Caron, G., Hansen, P., Jaumard, B.: The assignment problem with seniority and job priority constraints. Operations Research 47(3), 449-454 (1999)

9. Tseng, T.L., Huang, C.C., Chu, H.W., Gung, R.R.: Novel approach to multi-functional project team formation. International Journal of Project Management 22, 147-159 (2004)

10. Hadj-Hamou, K., Caillaud, E.: Cooperative design-A framework for a competency-based approach. In: 5th International Conference on IDMME (2004)

11. Fitzpatrick, E.L., Askin, R.G.: Forming effective worker teams with multi-functional skill requirements. Journal of Computers \& Industrial Engineering 48, 593-608 (2005)

12. Hlaoittinun, O., Bonjour, E., Dulmet, M.: A multidisciplinary team building method based on competency modeling in design project management. International Journal of Management Science and Engineering Management 3(3), 163-175 (2008)

13. Hlaoittinun, O.: Contribution à la constitution d'équipes de conception couplant la structuration du projet et le pilotage des compétences. PhD. Thesis, Université de Franche-Comté, France (2009)

14. Kirkpatrick, S., Gelatt, C.D., Vecchi, M.P.: Optimization by simulated annealing. Science 220(4598), 671-680 (1983)

15. Suman, B., Kumar, P.: A survey of simulated annealing as a tool for single and multiobjective optimization. Journal of the Operational Research Society 57(10), 1143-1160 (2006) 